\title{
Effect of Phacoemulsification on Intraocular Pressure Control in Primary Open Angle Glaucoma Previously Treated by Trabeculectomy: A Case-Control Study
}

\author{
Samir Aziz ${ }^{1 *}$, Nicky Spiers ${ }^{2}$, Jeffrey Jay ${ }^{3}$ \\ ${ }^{1}$ Moorfields Eye Hospital NHS Foundation Trust, London, England; ${ }^{2}$ Tennent Institute of Ophthalmology, Gartneval General Hospi- \\ tal, Glasgow, Scotland; ${ }^{3}$ University of Leicester, Leicester, England. \\ Email: "samiraziz@doctors.org.uk
}

Received December $26^{\text {th }}, 2011$; revised January $19^{\text {th }}, 2012$; accepted February $9^{\text {th }}, 2012$

\begin{abstract}
Purpose: To analyse the effect of phacoemulsification on the control of intraocular pressure in primary open angle glaucoma in patients having phacoemulsification after previous trabeculectomy and compare them with a control group who had trabeculectomy alone. Patients and Methods: Twenty one patients (one eye from each) who had phacoemulsification subsequent to trabeculectomy were identified, and compared with 41 controls. Intraocular pressure, bleb appearance, glaucoma medications, iris manipulation and complications were recorded. Each patient was followed for full 12 months. Failure of control was defined as follows: 1) intraocular pressure $>21 \mathrm{~mm} \mathrm{Hg}$ on medication, or 2) a greater number of glaucoma medications than before phacoemulsification. Results: The post operative change in intraocular pressure in the case group at 12 months was much less than that in the control $(p=0.001)$. The mean intraocular pressure had changed from $15.3 \mathrm{~mm} \mathrm{Hg}$ to $14.7 \mathrm{~mm} \mathrm{Hg}$. The control group showed an average intraocular pressure reduction of $6 \mathrm{~mm} \mathrm{Hg}$ at the last visit ( $\mathrm{p}>0.001)$. In phacoemulsification group, 19\% required 1 or 2 glaucoma medications at one year follow-up vs $19.5 \%$ in the control group. In phacoemulsification group, $9.5 \%$ showed flattening of a previously formed bleb at the last visit $(\mathrm{P}<0.001)$, compared with $9.7 \%$ of controls. Conclusions: The stability of glaucoma control in the first year after phacoemulsification in previously filtered eyes with primary open angle glaucoma is comparable to that of the natural course after trabeculectomy. The study is limited by the small number of cases available.
\end{abstract}

Keywords: Phacoemulsification; Trabeculectomy; Cataract; Glaucoma

\section{Introduction}

Glaucoma and cataract often coexist in the same eye $[1,2]$ not only because they both occur in the elderly population, $[3,4]$ but also because antiglaucoma medications may contribute to the formation and development of cataract [1]. In addition, glaucoma filtering surgery may accelerate cataract formation $[5,6]$, possibly in $14 \%$ $40 \%$ of patients $[7,8]$.

One of the accepted surgical options in patients with severe glaucoma and coexisting cataract is first to control the intraocular pressure (IOP) with trabeculectomy and then extract the cataract several months later [9]. Therefore, the management of visually significant cataract in a glaucoma patient who has had a previous trabeculectomy is a common clinical problem $[1,10,11]$.

Several studies have examined the effect of phacoemulsification (PE) $[1,10,11,12-14]$ and extracapsular cataract extraction (ECCE) $[1,10,11,12-13]$ on interme-

\footnotetext{
"Corresponding author.
}

diate and long-term IOP control after trabeculectomy, with conflicting results. When cataract surgery is performed in filtered eyes there is a risk of early postoperative increase in IOP and loss of long-term IOP control [3, 6,14-18]. Others have found that IOP control can be retained after cataract extraction by increasing antiglaucoma medications [10], while some report that increased IOP is not observed in patients with previous filtering surgery and that none of their patients required additional antiglaucoma medications [19].

More specifically, the effect of ECCE technique on filtering blebs has been investigated $[10,12,13]$. Ten to thirty-eight percent of eyes with previous trabeculectomy require additional medication or further glaucoma surgery to maintain IOP after ECCE with IOL implantation $[10,13,14]$. Phacoemulsification seems to have fewer adverse effects on the postoperative IOP control than ECCE; however, bleb dysfunction may still occur in the postoperative period $[10,13,15,18,20]$.

In this retrospective study we quantitatively analyze 
the effect of PE on the control of IOP in POAG in patients having $\mathrm{PE}$ after previous trabeculectomy (trabeculectomy-phacoemulsification group). In order to allow for any intrinsic instability of IOP control after trabeculectomy undisturbed by PE, we compare them with a control group who underwent trabeculectomy alone (trabeculectomy group).

\section{Patients and Methods}

A retrospective and consecutive case note review was performed on 21 Caucasian patients who had trabeculectomy for uncontrolled POAG followed by PE at Gartnavel General Hospital in Glasgow, UK. A trabeculectomyphacoemulsification (TP) group was compared with the control group (41 consecutive Caucasian patients) who had trabeculectomy (T) alone for uncontrolled POAG without cataract surgery at the same hospital. Patients with other types of glaucoma or who had received antimetabolites during or after the trabeculectomy were excluded. Each patient was followed for full 12 months after PE and trabeculectomy respectively. Only one eye of each patient was included.

The following data were obtained for each patient in both groups: gender, age, bleb appearance, number and type of glaucoma medications, previous ocular surgeries, time elapsed between $\mathrm{T}$ and PE, IOP preceding surgery, and IOP at 1 day, 1 week, 3, 6, 9, and 12 months after surgery, intraoperative iris manipulation (posterior synechialysis, stretching, sphincterotomies and iridectomy), intraoperative and postoperative complications, postoperative medications administered, and the dates when additional glaucoma medications were added. At the final visit, the number of glaucoma medications, IOP and bleb appearance were documented.

In the TP group, phacoemulsification was performed in 21 patients by several experienced surgeons, using a $3.2 \mathrm{~mm}$ superior clear-corneal incision. A foldable posterior chamber acrylic, hydrophobic lens (IOL) was inserted in the capsular bag. Small pupils were surgically enlarged by iris manipulation. In no case was an anterior chamber IOL inserted.

In all patients of both groups, same technique of $\mathrm{T}$ was performed by one surgeon (J.J.); using a fornix based conjunctival flap, however less than 5 were done by experienced surgeons who followed the same technique under his direct supervision. Post operative medications included topical corticosteroid and antibiotic drops used 4 times daily for 4 weeks in both groups. Cycloplegic drops were administered twice daily for 2 weeks after T. Intraocular pressure, bleb appearance, and number of glaucoma medications were compared between the 2 groups.
For the purposes of the study and for comparison with other studies, we used two criteria to define failure: 1) an IOP greater than $21 \mathrm{~mm} \mathrm{Hg}$ on medication, or 2) a greater number of glaucoma medications than before PE. Patients who had received antifibrotics were excluded.

Outcomes were compared between the two groups using the Mann-Whitney U test, Chi square and Student's t-test where appropriate. Random effects models with normal errors were fitted to the IOP profiles for months 1 , 3, 6, 9 and 12 using Proc Mixed in SAS. Because of great variability in pressures in the first month, measures taken prior to one month post-operatively were not modeled. Models were compared using the Likelihood Ratio Test. Differences were considered significant at the $5 \%$ level.

\section{Results}

The patient's characteristics are shown in Table 1. There is a difference between the mean ages in the 2 groups. The mean time between T and PE was 52.6 months (SD 22.6, range 16 - 93). In the TP group, $9.5 \%(n=2$, Table 2) showed flattening of a previously formed bleb at one year follow-up $(\mathrm{P}<0.03)$. The trabeculectomy group was similar $(9.7 \%, \mathrm{n}=4)$.

The mean IOP of the case group one year after PE had fallen very slightly from $15.3 \mathrm{~mm} \mathrm{Hg}$ (SD 4.5 , range 10 25) preoperatively to $14.7 \mathrm{~mm} \mathrm{Hg}$ (SD 3.5, range 10 22). However, one patient had an IOP of $22 \mathrm{~mm} \mathrm{Hg}$ at the latest examination. At the last visit, patients who required antiglaucoma medications after PE had a mean IOP before PE of $21.5 \mathrm{~mm} \mathrm{Hg}$ (SD 1.7, range 20 - 23), which was higher than those controlled without glaucoma medication $14 \mathrm{~mm} \mathrm{Hg}$ (SD 3.9, range 10 - 25).

The post operative change in IOP in the TP group at 12 months was much less than the change in IOP following trabeculectomy (Median change 0 versus $-6 \mathrm{p}=$ 0.001; Mann-Whitney U test).

The control group showed an average IOP reduction of $6 \mathrm{~mm} \mathrm{Hg}$ in the last visit $(\mathrm{p}>0.001)$. There was no evidence that the difference in mean IOP between the groups varied with time, or that there was any change in between subjects variation in pressure. The best fitting model had parallel quadratic mean profiles for the case and control groups, with an increase in mean IOP over time (Figures 1 and 2). Figure 1 also confirms that the curves for long term change in IOP are similar in both groups.

The estimated mean IOP profile shown in Figure $\mathbf{1}$ is higher for cases than for controls, but this difference was not significant (mean difference $0.85 \mathrm{~mm} \mathrm{Hg}$; 95\% CI -0.84 to 2.54$)$.

Two outliers in the control group were not well fitted 
Table 1. Characteristics of patients in the two groups.

\begin{tabular}{|c|c|c|c|c|c|}
\hline & & Trabeculectomy & $\begin{array}{l}\text { Trabeculectomy- } \\
\text { Phacoemulsification }\end{array}$ & P-value & Test \\
\hline Diagnosis & POAG & 41 & 21 & & \\
\hline Age (years) & Mean (range) & $74(57-87)$ & $78(61-91)$ & 0.01 & Unpaired t-test \\
\hline \multirow[t]{3}{*}{ Sex } & & & & 0.24 & Chi square \\
\hline & Male & 20 & 7 & & \\
\hline & Female & 21 & 14 & & \\
\hline \multirow[t]{2}{*}{ IOP (mean) } & Baseline & $15.3(10-25)$ & $14.7(10-22)$ & & \\
\hline & Postoperative & $21(15-31)^{*}$ & $15(7-26) \dagger$ & & \\
\hline $\begin{array}{l}\text { Elapsed time between glaucoma and } \\
\text { cataract surgeries (months) }\end{array}$ & Mean (range) & N/A & $52.6(16-93)$ & & \\
\hline Iris manipulation & & & 5 & & \\
\hline
\end{tabular}

POAG: primary open angle glaucoma; IOP: intraocular pressure; *: 12 months post trabeculectomy; $\uparrow: 12$ months post phacoemulsification.

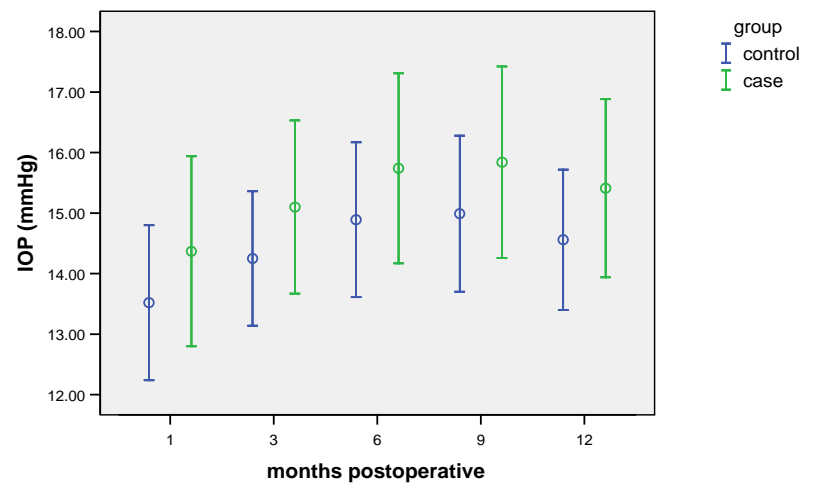

Figure 1. Predicted mean intraocular pressure from final model, with $95 \%$ confidence intervals.

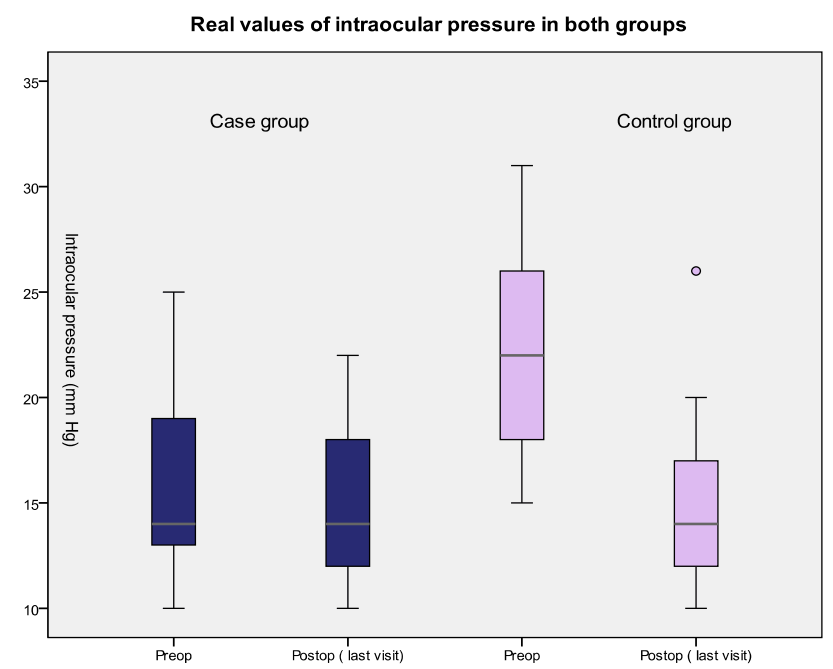

Figure 2. Real values of intraocular pressure in both groups. by the model. ID 121 had unusually high IOP throughout. ID 140 had an unusual increase in IOP from 6 at month 1 to 22 at month 3 and persisted at that level. When the final model was refitted with these two subjects included there was a slight increase in the difference in means between groups (Table 3), but the difference in mean profiles between the groups did not reach significance in either model.

There was one missing value at month 9 in the case group. The other values for this individual were included in the analysis, and the missing value is not considered to have any bearing on the conclusions.

In the PE group, 4 patients (19\%) required 1 or 2 glaucoma medications at one year (Table 2). One had high IOP preoperatively; the other had required treatment prior to PE. In the trabeculectomy group eight patients (19.5\%) used glaucoma medication one year after $\mathrm{T}$, and 5 of them used a single medication. Iris manipulation was required in 5 patients ( 3 posterior synechialysis, 1 stretching, and 1 sphincterotomy) to disrupt posterior synechiae during cataract surgery in the PE group; three of these needed glaucoma medications in the first month. However, IOP was controlled thereafter without lowering drops.

In the TP group there were postoperative IOP spikes in 8 eyes $(38 \%)$ on the first day and in a further 2 eyes in the first week. In the same group, endophthalmitis developed in one eye one week postoperatively, it was successfully treated with intravitreal amikacin, it retained a VA (visual acuity) of 6/60 in the last visit.

\section{Discussions}

Cataract extraction in eyes with a functioning filtering 
Table 2. Intraocular pressure, number of medications and bleb appearance in the case group.

\begin{tabular}{|c|c|c|c|c|c|}
\hline \multirow{2}{*}{ Case } & \multicolumn{2}{|c|}{ IOP (mm Hg) } & \multicolumn{2}{|c|}{ Number of medications } & \multirow{2}{*}{ Bleb appearance } \\
\hline & Preop & Last visit & Preop & Last visit & \\
\hline 1 & 14 & 16 & 0 & 0 & $\mathrm{NC}$ \\
\hline 2 & 14 & 12 & 0 & 0 & $\mathrm{NC}$ \\
\hline 3 & 19 & 12 & 0 & 0 & $\mathrm{NC}$ \\
\hline 4 & 10 & 12 & 0 & 0 & $\mathrm{NC}$ \\
\hline 5 & 14 & 13 & 0 & 0 & $\mathrm{NC}$ \\
\hline 6 & 13 & 18 & 0 & 0 & $\mathrm{NC}$ \\
\hline 7 & 12 & 19 & 0 & 0 & $\mathrm{NC}$ \\
\hline 8 & 11 & 18 & 0 & 0 & $\mathrm{NC}$ \\
\hline 9 & 20 & 14 & 1 & 1 & $\mathrm{NC}$ \\
\hline 10 & 14 & 12 & 0 & 0 & $\mathrm{NC}$ \\
\hline 11 & 13 & 14 & 0 & 0 & $\mathrm{NC}$ \\
\hline 12 & 20 & 10 & 1 & 1 & Flattened \\
\hline 13 & 23 & 22 & 0 & 1 & Flattened \\
\hline 14 & 14 & 12 & 0 & 0 & $\mathrm{NC}$ \\
\hline 15 & 10 & 19 & 0 & 0 & $\mathrm{NC}$ \\
\hline 16 & 15 & 17 & 0 & 0 & $\mathrm{NC}$ \\
\hline 17 & 10 & 10 & 0 & 0 & $\mathrm{NC}$ \\
\hline 18 & 25 & 12 & 0 & 0 & $\mathrm{NC}$ \\
\hline 19 & 23 & 12 & 0 & 2 & $\mathrm{NC}$ \\
\hline 20 & 14 & 15 & 0 & 0 & $\mathrm{NC}$ \\
\hline 21 & 14 & 20 & 0 & 0 & $\mathrm{NC}$ \\
\hline
\end{tabular}

IOP: intraocular pressure; NC: no change.

Table 3. Mean difference in intra ocular pressure (IOP) profiles from random effects model.

\begin{tabular}{cccccc}
\hline Model & $\mathbf{N}$ & Difference in mean IOP (Case-control) & LCL & UCL & P-value for difference \\
\hline 1 & 62 & 0.77 & -0.99 & 2.53 & 0.39 \\
2 (Outliers excluded) & 60 & 1.05 & -0.54 & 2.64 & 0.20 \\
\hline
\end{tabular}

N: number; IOP: intraocular pressure; LCL: lower 95\% confidence limit; UCL: upper 95\% confidence limit.

bleb is considered to be a risk [17]. Small incision cataract surgery is the technique of choice in this group [1, 16], because there is less conjunctival dissection and inflammation [1]. Several studies report that PE has a minimal effect on the long-term mean IOP after $\mathrm{T}[2,10$, 12]. However, PE may jeopardize a previously functioning filtering bleb and result in increase in IOP $[4,11$, $15,18]$.

This study is an attempt to isolate the IOP as one specific aspect of glaucoma and to determine if that single factor is affected by later PE.
We did not include visual field since it might be influenced by lens opacities [21]. We showed that the IOP in the TP group at up to one year after PE was not significantly altered. Other studies (Table 4) have shown variable results and in some, either no significant difference or a decrease in IOP was detected [10,11,14,22,23]. Park et al. [14] used a control group that had T alone and showed that PE seemed to have no effect on IOP control after PE. However, unlike our study their case-control study was not limited to cases of POAG. Furthermore, antimetabolites had been used which might have affected 
Table 4. Summary of studies on the effects of phacoemulsification in eyes with filtering blebs.

\begin{tabular}{|c|c|c|c|c|}
\hline Study & No. & Follow-up (m) & Success (\%) & Definition of success \\
\hline Seah et al. [13] & 6 & 13.6 & 67 & $\begin{array}{l}\text { IOP }<19 \mathrm{~mm} \mathrm{Hg} \text { with no additional surgery and no additional } \\
\text { medications }\end{array}$ \\
\hline Chen et al. [10] & 57 & 17.6 & 74 & No additional medications, bleb needling, or glaucoma surgery \\
\hline Park et al. [14] & 40 & 20.1 & $80(3 \mathrm{yrs})$ & $\begin{array}{l}\text { No greater number of medications: IOP } \leq 21 \mathrm{~mm} \mathrm{Hg} \text { or }>20 \% \text { re- } \\
\text { duction on } 2 \text { consecutive visits compared with pretrabeculectomy }\end{array}$ \\
\hline Manoj et al. [11] & 21 & 15.1 & 100 & IOP $<18 \mathrm{~mm} \mathrm{Hg}$ and within the target pressure \\
\hline Crichton \& Kirker [15] & 69 & 23.2 & 77 & $\begin{array}{l}\text { No additional surgery and no additional medications compared } \\
\text { with pre cataract }\end{array}$ \\
\hline Rebolleda \& Munoz-Negrete. [17] & 49 & 19.5 & 67.4 & No glaucoma medications, surgery, or bleb needling to control IOP \\
\hline Ehrnrooth et al. [4] & 46 & 25.3 & 69.5 & $\begin{array}{l}\text { IOP } \leq 21 \mathrm{~mm} \mathrm{Hg} \text { with no additional surgery and no or single } \\
\text { topical medications }\end{array}$ \\
\hline Present study & 21 & 12 & 85.7 & $\begin{array}{l}\mathrm{IOP} \leq 21 \mathrm{~mm} \mathrm{Hg} \text { with no additional surgery and no additional } \\
\text { medications }\end{array}$ \\
\hline
\end{tabular}

the outcome, in contrast to our study where patients who had received antimetabolites were excluded in order to reduce case mix and subsequently bias.

On the other hand, the most frequent conclusion is that there is an increase in IOP after PE [1,10,12,15,17,19, 23-25].

Our aim was to describe the changes provoked by a second surgical procedure. When the time between trabeculectomy and PE was greater than 1 year the interval between surgeries had no effect on bleb failures, IOP, or medication changes [17]. In our study cohort, the time between $\mathrm{T}$ and $\mathrm{PE}$ is rather long, since we feel that most of them do not need cataract surgery earlier than that.

The success rate after 1 year in our TP group was $85.7 \%$, Table 4 shows the success rate reported in the literature. The rate varies between $67 \%$ and $100 \%$ but the definition of success is slightly different in the different studies and not all included a control group. Most of the failures occurred between 6 and 24 months after PE, indicating that the effect on bleb filtration is a delayed response [26].

There is a recognized trend for a late rise in IOP after $\mathrm{T}$ [18]; therefore PE might not be the only possible adverse influence on IOP [27]. A decrease in IOP over time after successful $\mathrm{T}$ has been reported [28]. In our study, allowing for the possible change in long term IOP control after T, PE seemed to have no additional effect on IOP control.

The use of glaucoma medications is similar in the case (19\%) and control (19.5\%) groups. This figure matches one other study $(20 \%)$ [15]. But most authors report more frequent use of medications [10,14]; (22\%) [29], (34.7\%) [17], (49.4\%) [2], (41\%) [4]. In our study, the number of glaucoma medications did not change much in the TP group during the course of the study, whereas in the $\mathrm{T}$ group $9 \%$ required additional pressure-lowering medications.

Evaluation of bleb morphology in retrospective studies without standardized criteria is difficult and very subjective. We found flattening of the bleb in $9.5 \%$ and $9.7 \%$ in the case and control groups respectively. Others have found this to be more frequent $(77.6 \%)$ [17], (18\%) [10].

It is likely that the inflammatory response elicited by surgery induces subconjunctival scarring [20]. This could explain the flattening of the filtering bleb and subsequent IOP increase that may occur even after the relatively atraumatic PE procedure [10,12,13,17,29]. The presence of a functional filtering bleb before surgery does not guarantee long-term IOP control after PE [17].

In our study, PE was performed at least 16 months after $\mathrm{T}$, and under these conditions, the time between $\mathrm{T}$ and $P E$ did not seem to influence failure, glaucoma medication use or IOP changes. Some authors believe that the filtering bleb needs sufficient time ( $>5$ months) [14], ( $\geq 6$ months) [24], or ( $\geq 1$ year) [13] to develop properly since the inflammation associated with cataract surgery may induce bleb failure [10]. However, others showed no association between IOP control and the timing of cataract surgery [14].

Iris manipulation in our study seemed not to be associated with poor IOP control, bleb failure or need for additional medications. Similar findings were reported by others good IOP control [18,27], no bleb failure [10,13] no additional medications [15,16,24]. Other studies did, however, find an association with bleb failure [24,27].

Severe postoperative complications after PE were rare in our study. Early postoperative IOP spikes are frequently observed after cataract surgery in glaucomatous eyes. We observed an IOP spike of $\geq 8 \mathrm{~mm} \mathrm{Hg}$ in 4 eyes (19\%) 1 day after PE. A prospective study by Rebolleda 
\& Muñoz-Negrete [17] found a similar rate $(18.4 \%$, IOP $>10 \mathrm{~mm} \mathrm{Hg})$. Others have reported higher (57\%) [30], $(50 \%)[14],(37 \%)[14]$, or lower rates $(6.3 \% \geq 30 \mathrm{~mm}$ $\mathrm{Hg})[24]$.

Intraocular pressure fluctuations during the first postoperative months after routine cataract extraction are well known [11].

Our study used only eyes with POAG, which carries the best prognosis for T. Therefore, our favorable observations may not be extrapolated to other types of glaucoma where a successful drainage fistula might more readily be compromised by subsequent $\mathrm{PE}$.

There is 4 year age difference between both groups; however we do not feel that this is a source of clinically meaningful bias. Our study has the benefit of a control group which enables us to make allowance for the change in IOP which might occur after T. Another strength is the analysis of repeated measurements of IOP during the year which allowed a detailed comparison of the behavior of the IOP in the two groups.

Limitations of this study are its retrospective nature and the lack of statistical significance; this may be attributable to the relatively small sample size.

We conclude that the stability of glaucoma control in the first year after PE in previously filtered eyes with POAG is comparable to that of the natural course of T.

A future, large, prospective and controlled study could provide more reliable data about the effect of $\mathrm{PE}$ on the function of a previous fistulising operation.

\section{REFERENCES}

[1] D. Halikiopoulos, M. R. Moster, A. Azuara-Blanco, R. P. Wilson, C. M. Schmidt, G. L. Spaeth, L. J. Katz and J. J. Augsburger, "The Outcome of the Functioning Filter after Subsequent Cataract Extraction," Ophthalmic Surgery and Lasers, Vol. 32, No. 2 , 2001, pp. 108-117.

[2] D. S. Friedman, H. D. Jampel, L. H. Lubomski, J. H. Kempen, H. Quigley, N. Congdon, H. Levkovitch-Verbin, K. A. Robinson and E. B. Bass, "Surgical Strategies for Coexisting Glaucoma and Cataract-An Evidence-Based Update," Ophthalmology, Vol. 109, No. 10, 2002, pp. 19021913. doi:10.1016/S0161-6420(02)01267-8

[3] S. A. Obstbaum, "Glaucoma and Intraocular Lens Implantation," Journal of Cataract and Refractive Surgery, Vol. 12, No. 3, 1986, pp. 257-261.

[4] P. Ehrnrooth, I. Lehto, P. Puska and L. Laatikainen, "Phacoemulsification in Trabeculectomized Eyes," Acta Ophthalmologica Scandanavia, Vol. 83, No. 5, 2005, pp. 561-566. doi:10.1111/j.1600-0420.2005.00499.x

[5] F. D'Ermo, L. Bonomi and D. Doro, "A Critical Analysis of the Long-Term Results of trabEculectomy," American Journal of Ophthalmology, Vol. 88, No. 5, 1979, pp. 829835.

[6] K. B. Mills, "Trabeculectomy: A Retrospective Long-
Term Follow-Up of 444 Cases," British Journal of Ophthalmology, Vol. 65, No. 11, 1981, pp. 790-795. doi:10.1136/bjo.65.11.790

[7] P. G. Watson, C. Jakeman, M. Oztur, M. F. Barnett, F. Barnett and K. T. Khaw, "The Complications of Trabeculectomy (a 20 Year Follow-Up)," Eye, Vol. 4, No. 3, 1990, pp. 425-438. doi:10.1038/eye.1990.54

[8] A. C. B. Molteno, N. J. Bosma and J. M. Kittleson, "Otago Gluacoma Surgery Outcome Study: Long-Term Results of Trabeculectomy-1976 to 1995," Ophthalmology, Vol. 106, No. 9, 1999, pp. 1742-1750. doi:10.1016/S0161-6420(99)90351-2

[9] M. Sheilds, "Another Reevaluation of Combined Cataract and Glaucoma Surgery," American Journal of Ophthalmology, Vol. 115, No. 6, 1993, pp. 806-811.

[10] P. P. Chen, Y. K. Weaver, D. L. Budenz, W. J. Feuer and R. K. Parrish II, "Trabeculectomy Function after Cataract Extraction," Ophthalmology, Vol. 105, No. 10, 1998, pp. 1928-1935. doi:10.1016/S0161-6420(98)91044-2

[11] B. Manoj, D. Chako and M. Y. Khan, "Effect of Extracapsular Cataract Extraction and Phacoemulsification Performed after Trabeculectomy on Intraocular Pressure," Journal of Cataract \& Refractive Surgery, Vol. 26, No. 1, 2000, pp. 75-78. doi:10.1016/S0886-3350(99)00321-1

[12] M. A. Dickens and L. F. Cashwell, "Long-Term Effect of Cataract Extraction on the Function of an Established Filtering Bleb," Ophthalmic Surgery \& Lasers, Vol. 27, No. 1, 1996, pp. 9-14.

[13] S. K. Seah, A. Jap, J. A. Prata, G. Baerveldt, P. P. Lee, D. K. Heuer and D. S. Minckler, "Cataract Surgery after Trabeculectomy," Ophthalmic Surgery \& Lasers, Vol. 27, No. 7, 1996, pp. 587-594.

[14] H. J. Park, Y. H. Kwon, M. Weitzman and J. Caprioli, "Temporal Corneal Phacoemulsification in Patients with Filtered Glaucoma," Archives of Ophthalmology, Vol. 115, No. 11, 1997, pp. 1375-1380. doi:10.1001/archopht.1997.01100160545003

[15] A. C. S. Crichton and A. W. Kirker, "Intraocular Pressure and Medication Control after Clear Corneal Phacoemulsification and Acrysof Posterior Chamber Intraocular Lens Implantation in Patients with Filtering Blebs," Journal of Glaucoma, Vol. 10, No. 1, 2001, pp. 38-46. doi:10.1097/00061198-200102000-00008

[16] R. J. Casson, C. E. Riddell, R. Rahman, D. Byles and J. F. Salmon, "Long-Term Effect of Cataract Surgery on Intraocular Pressure after Trabeculectomy-Extra Extraction Versus Phacoemulsification," Journal of Cataract \& Refractive Surgery, Vol. 28, No. 1, 2002, pp. 2159-2164. doi:10.1016/S0886-3350(02)01501-8

[17] G. Rebolleda and F. J. Muñoz-Negrete, "Phacoemulsification in Eyes with Functioning Filtering Blebs: A Prospective Study," Ophthalmology, Vol. 109, No. 12, 2002, pp. 2248-2255. doi:10.1016/S0161-6420(02)01246-0

[18] J. Klink, B. Schmitz, W. E. Lieb, T. Klink, H. J. Grein, J. Sold-Darseff, A. Heinold and F. Grehn, "Filtering Bleb Function after Clear Cornea Phacoemulsification: A Prospective Study," British Journal of Ophthalmology, Vol. 89, No. 5, 2005, pp. 597-601. 


\section{doi:10.1136/bjo.2004.041988}

[19] K. S. Kooner, D. D. Dulaney and T. J. Zimmerman, "Intraocular Pressure Following Ecce and Iol Implantation in Patients with Glaucoma," Ophthalmic Surgery, Vol. 19, No. 8, 1988, pp. 570-575.

[20] T. K. Sharma, S. Arora and P. G. Corridan, "Phacoemulsification in Patients with Previous Trabeculectomy: Role of 5-Flourouracil," Eye, Vol. 21, No. 6, 2007, pp. 780783. doi: $10.1038 /$ sj.eye. 6702327

[21] G. S. Ang, M. Shunmugam and A. Azuara-Blanco, "Effect of Cataract Extraction on the Glaucoma Progression Index (GPI)," Journal of Glaucoma, Vol. 19, No. 4, 2010, pp. 275-278.

[22] R. T. Oyakawa and A. E. Maumenee, "Clear Cornea Cataract Extraction in Eyes with Functioning Bleb," American Journal of Ophthalmology, Vol. 93, No. 3, 1982, pp. 294-298.

[23] W. Doyle and M. F. Smith, "Effect of Phacoemulsification Surgery on Hypotony Following Trabeculectomy Surgery," Archives of Ophthalmology, Vol. 118, No. 6, 2000, pp. 763-765.

[24] A. Derbolav, C. Vass, R. Menapace, K. Schmetterer and A. Wedrich, "Long-Term Effect of Phacoemulsification on Intraocular Pressure after Trabeculectomy," Journal of Cataract \& Refractive Surgery, Vol. 28, No. 3, 2002, pp. 425-430. doi:10.1016/S0886-3350(01)01189-0

[25] X. Wang, H. Zhang, S. Li and N. Wang, "The Effects of Phacoemulsification on Intraocular Pressure and Ultra- sound Biomicroscopic Image of Filtering Bleb in Eyes with Cataract and Functioning Filtering Blebs," Eye, Vol. 23, No. 1, 2009, pp. 112-116. doi:10.1038/sj.eye.6702981

[26] R. Casson, R. Rahman and J. F. Salmon, "Phacoemulsification with Intraocular Lens Implantation after Trabeculectomy," Journal of Glaucoma, Vol. 11, No. 5, 2002, pp. 429-433. doi:10.1097/00061198-200210000-00011

[27] L. B. Cantor, A. Mantravadi, D. WuDunn, K. Swamynathan and A. Cortes, "Morphologic Classification of Filtering Blebs after Glaucoma Filtration Surgery: The Indiana Bleb Appearance Grading Scale," Journal of Glaucoma, Vol. 12, No. 3, 2003, pp. 266-271. doi:10.1097/00061198-200306000-00015

[28] Y. H. Kwon, C. S. Kim, M. B. Zimmerman, W. L. Alward and S. S. Hayreh, "Rate of Visual Loss and Long-Term Visual Outcome in Primary Open-Angle Glaucoma," American Journal of Ophthalmology, Vol. 132, No. 1, 2001, pp. 47-56. doi:10.1016/S0002-9394(01)00912-6

[29] J. F. Murchison and M. B. Sheilds, "An Evaluation of Three Surgical Approaches for Coexisting Cataract and Glaucoma," Ophthalmic Surgery, Vol. 20, No. 6, 1989, pp. 393-398.

[30] J. F. Bigger and B. Becker, "Cataracts and Primary Open Angle Glaucoma: The Effect of Uncomplicated Cataract Extraction on Glaucoma Control," Transactions of the American Academy of Ophthalmology and Otolaryngology, Vol. 75, No. 2, 1971, pp. 260-272. 